\title{
507.
}

\section{ON THE MECHANICAL DESCRIPTION OF CERTAIN QUARTIC CURVES BY A MODIFIED OVAL CHUCK.}

[From the Proceedings of the London Mathematical Society, vol. IV. (1871-1873), pp. 186-190. Read December 12, 1872.]

THE geometrical principle of the oval chuck is the well-known one that if a plane move in such manner that two lines $O x, O y$, fixed in the plane and moveable with it, pass through two fixed points $A, B$, respectively, then any fixed point $P$ traces out on the plane an ellipse. The point $A$ is on the (geometrical) axis of the mandril; there is connected with the head a guide-ring moving horizontally; the point $B$ is the centre of the guide-ring, this being a ring connected with the head, moveable horizontally at right angles to the axis in such wise that the distance $A B$ of the two centres is adjustable to any given value; the fixed point $P$ is the tool, which practically is held on the level of the axis, that is, at a point in the line $A B$. The guide-ring remains fixed during the motion of the lathe.

It occurred to me that a chuck applicable to ornamental turning might be constructed by giving to the guide-ring a reciprocating motion synchronous with the rotation of the mandril; viz. for this purpose it is only necessary to affix to the axis of the mandril an eccentric, working in a frame attached to the guide-ring so as to move the centre $B$ of the guide-ring backwards and forwards along the line $A B$ : the curve is thus that described by the fixed point $P$ upon a plane moving in such manner that the lines $O x, O y$ pass always through the points $A, B$ respectively; the former of these being a fixed point, the latter of them a point moving according to determinate law backwards and forwards along a fixed line through $A$.

The plan is carried out in a drawing apparatus which I have had constructed in wood, the axis being here vertical instead of horizontal, and the details of course different from what they would be for a lathe. 
The apparatus consists of a piece of inch-board, about 10 inches long by 7 inches broad, pierced with a circular hole of 1 inch diameter for a vertical axis: the edges of the board serve as guides for the frame $L$, which carries the guide-ring, and resting on the board we have the frame $M$, itself guided by the frame $L$ : the two frames move independently of each other, but they can be clamped together; the axis has
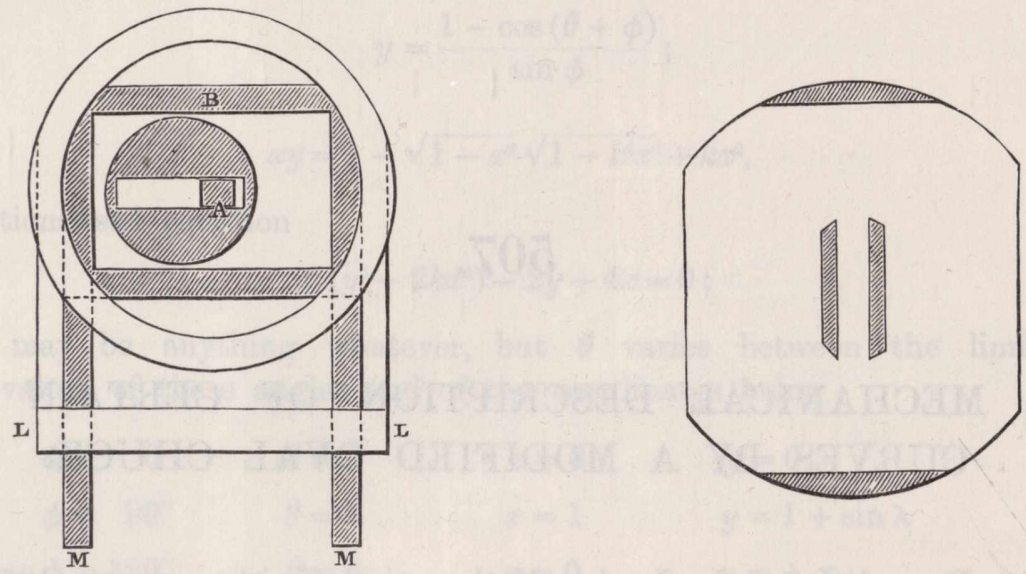

upon it a square nut, the sides of which work in the slot of an eccentric, the throw of this being adjustable by means of a screw passing through the nut and axis, and there is above the eccentric a square nut shown in the figure. This is capable of rotation round the axis, so that two of its sides may be placed either parallel with or inclined to the sides of the slot; but I fix it with two sides parallel to those of the slot by means of a screw run into the axis. The upper surfaces of the lastmentioned nut and of the guide-ring are flush with each other; and we then have a table or bed having, on its under-surface, guides which work on the outer edges of the guide-ring and on two edges of the nut. It will be observed that the bed may be placed in two different positions, viz. the guides may work on either pair of edges of the nut, those which are parallel to the sides of the slot, or those which are at right angles to it.

Supposing the bed placed as above upon the guide-ring and nut, then if the frames $L$ and $M$ are disconnected, and the former of them is fixed, the frame $M$ will, on rotation of the axis, be carried backwards and forwards by the eccentric, but this will in no wise affect the motion of the bed; the arrangement is then equivalent to the oval chuck, and a pencil fixed above the bed in any given position will trace out upon it an ellipse. If, however, the frame $L$, instead of being fixed, is clamped to the frame $M$, then the two frames, and therefore the guide-ring, are carried backwards and forwards by the eccentric, and the curve traced out by the pencil is no longer an ellipse; it is, as I proceed to show, a special form of trinodal quartic; viz. there is a tacnode (=two nodes) at infinity, and a third node, which may be a crunode, cusp, or acnode. In the last-mentioned case, the acnode or conjugate point is, as usual, not exhibited by the mechanical description, and the curve has no visible singularity. 
Let the coordinates of the fixed point $P$, referred to axes through $A$, the first of them perpendicular to, and the second coincident with, $A B$, be $b, c$; let the distance $A B$ be $=a$; and let $\theta$ denote the angle $B A O$ : then, if $x, y$ are the coordinates of $P$ referred to the origin $O$ and axes $O x, O y$, we have

$$
\begin{aligned}
x+a \cos \theta & =b \sin \theta+c \cos \theta, \\
y \quad & -b \cos \theta+c \sin \theta,
\end{aligned}
$$

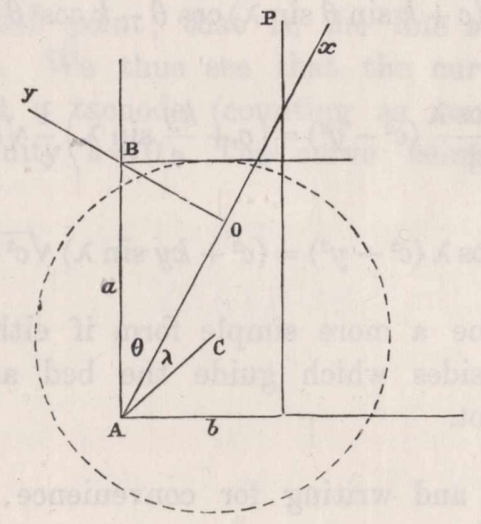

which, if $a$ be constant, gives a quadric equation, or the curve is an ellipse; and, in particular, if $b=0$, that is if the point $P$ is on the line $A B$, then we have

or the curve is

$$
x=(c-a) \cos \theta, \quad y=c \sin \theta,
$$

$$
\frac{x^{2}}{(c-a)^{2}}+\frac{y^{2}}{c^{2}}=1
$$

But if $a$ is a given function of $\theta$, then the equation is still found by eliminating $\theta$ between the two equations for $x$ and $y$. In particular, if the distance $A B$ is given as the perpendicular upon the tangent of a circle, as shown in the figure, then if $k$ be the radius $A C$ of this circle, and $\lambda$ the inclination of $A C$ to $A x$ ( $k$ and $\lambda$ being taken to be constants), we have

and the equations are

$$
a=k \cos (\theta+\lambda),
$$

$$
\begin{aligned}
& x=b \sin \theta+\{c-k \cos (\theta+\lambda)\} \cos \theta, \\
& y=-b \cos \theta+c \sin \theta .
\end{aligned}
$$

The elimination is nearly the same as if $b$ were $=0$; viz. we may determine $\gamma, \alpha$ in such wise that

$$
\begin{aligned}
b \sin \theta+c \cos \theta=\gamma \cos (\theta+\alpha), & =\gamma \cos \phi \quad \text { suppose, } \\
-b \cos \theta+c \sin \theta & =\gamma \sin (\theta+\alpha),=\gamma \sin \phi
\end{aligned}
$$

C. VIII. 
and then

$$
\begin{aligned}
& x=\gamma \cos \phi-k \cos (\phi+\lambda-\alpha) \cos (\phi-\alpha), \\
& y=\gamma \sin \phi .
\end{aligned}
$$

I will, for greater simplicity, at once write $b=0$ : the equations thus are

$$
\begin{aligned}
& x=(c-k \cos \theta \cos \lambda+k \sin \theta \sin \lambda) \cos \theta, \\
& y=c \sin \theta ;
\end{aligned}
$$

or say the first is

$$
x=(c+k \sin \theta \sin \lambda) \cos \theta-k \cos ^{2} \theta \cos \lambda ;
$$

whence we have

$$
x+\frac{k \cos \lambda}{c^{2}}\left(c^{2}-y^{2}\right)=\left(c+\frac{k y}{c} \sin \lambda\right) \frac{1}{c} \sqrt{c^{2}-y^{2}},
$$

that is

$$
c^{2} x+k \cos \lambda\left(c^{2}-y^{2}\right)=\left(c^{2}+k y \sin \lambda\right) \sqrt{c^{2}-y^{2}},
$$

an equation which will assume a more simple form if either $\lambda=0$ or $\lambda=90^{\circ}$; that is, if in the apparatus the nut-sides which guide the bed are either at right angles, or parallel, to the sides of the slot.

Taking the general case, and writing for convenience $\cos \theta=\xi$, $\sin \theta=\eta$, the curve is given by equations of the form

$$
\begin{gathered}
x=(\xi, \eta, 1)^{2}, \\
y=(\xi, \eta, 1)^{2}, \\
\xi^{2}+\eta^{2}=1 ;
\end{gathered}
$$

viz. the elimination of $\xi, \eta$ from these equations leads to the equation of the curve. The points of the curve have thus a $(1,1)$ correspondence with those of the circle $\xi^{2}+\eta^{2}=1$; or, the circle being unicursal, the curve is also unicursal. Moreover, considering the intersections of the curve with an arbitrary line $a x+b y+c=0$, the points of intersection correspond to the points of intersection of the circle by the quadric $a(\xi, \eta, 1)^{2}+b(\xi, \eta, 1)^{2}+c=0$; viz. there are four points of intersection, or the curve is a quartic, and hence it is a binodal quartic. But it is a binodal quartic of a special form: to show this more clearly, I introduce for homogeneity the coordinates $z, \zeta$, so that the foregoing equations become

$$
x: y: z=(\xi, \eta, \zeta)^{2}:(\xi, \eta, \zeta)^{2}: \zeta^{2} \text {, where } \xi^{2}+\eta^{2}-\zeta^{2}=0 ;
$$

the curve corresponding to these equations is, as just seen, a binodal quartic. But in the case in hand the form is the more special one,

$$
x: y: z=(\xi, \eta, \zeta)^{2}: \xi \zeta \quad: \zeta^{2} \text {, where } \xi^{2}+\eta^{2}-\zeta^{2}=0 .
$$

The intersections by the arbitrary line $a x+b y+c z=0$ are the points corresponding to the intersections of the circle $\xi^{2}+\eta^{2}-\zeta^{2}=0$ by the quadric $a(\xi, \eta, \quad \zeta)^{2}+b \xi \zeta+c \zeta^{2}=0$, 
giving four intersections. But the intersections by the line $b y+c z=0$ (that is, by any line through the point $y=0, z=0)$ are obtained from the equation $b \xi \xi+c \zeta^{2}=0$; viz. this breaks up into $b \xi+c \zeta=0, \zeta=0$, and the last factor combined with the equation of the circle gives $\zeta=0, \xi^{2}+\eta^{2}=0$, the two circular points at infinity, corresponding each to the point $y=0, z=0$ : the other factor gives points corresponding to two variable points on the curve; that is, a line through the point $y=0, z=0$ meets the curve in this point twice and in two other points. Again, making $b=0$, or taking the line to be the line at infinity $z=0$, the equations then are $\zeta^{2}=0, \xi^{2}+\eta^{2}=0$; viz. we then have the circular points at infinity each twice, corresponding to the point $y=0$, $z=0$ four times, and no other point; that is, the line $z=0$ meets the curve in the point $y=0, z=0$ four times. We thus see that the curve has at $y=0, z=0$, that is at infinity on the line $y=0$, a tacnode (counting as two nodes), the tangent at this point being the line at infinity $z=0$. The curve being trinodal has of course one other node. 